\title{
Monitoramento da Integridade Física de Cabos Ópticos de Telecomunicações Usando um Sistema Modalmétrico no Domínio do Tempo
}

\begin{abstract}
Ricardo M. Ribeiro, Yanes C. Balod, Andrés P. I.
Resumo - Este trabalho descreve pela primeira vez a resposta temporal de um sensor modalmétrico à fibra óptica para perturbações de contato mecânico aplicado ao monitoramento sensível da integridade física de cabos ópticos de Telecomunicações. Tal monitoramento é impossível de ser feito por Refletometria Óptica no Domínio do Tempo (OTDR) tradicional. O mecanismo do sistema modalmétrico é baseado na interferência entre os vários modos propagantes, que acontece na emenda entre fibras monomodo (SM) padrão e multimodo de índice gradual (GI-MM). O dispositivo é extremamente sensível a um contato mecânico de baixa magnitude, mas não a variação de temperatura, ambos aplicados pontualmente.
\end{abstract}

Palavras-chaves - Sensor modalmétrico, fibra óptica multimodo, perturbação, interferômetro, integridade física, cabo óptico.

\begin{abstract}
This work describe for the first time the temporal response of a fibre optic modalmetric sensor for mechanical disturbance intended for use in integrity monitoring of Telecommunications optical cables. The usual Optical Time-Domain Reflectometry (OTDR) is unsuitable for such monitoring. The physical mechanism behind the modalmetric sensor is based on the interference among the modes at the splice between the single-mode (SM) and the gradedindex multimode (GI-MM) fibre. The device has shown high sensitivity under very weak disturbances but none response under temperature variations.
\end{abstract}

Keywords - Modalmetric sensor, multimode optical fibre, disturbance, interferometer, physical integrity, optical cable.

Ricardo M. Ribeiro, rmr@pq.cnpq.br, Yanes $\quad$ C. Balod, yanes@ pobox.com, Murilo B. de Carvalho, murilo@telecom.uff.br Andrés P. L. Barbero, Pablo@telecom.uff.br, Laboratório de Comunicações Ópticas, Departamento de Engenharia de Telecomunicações, Universidade Federal Fluminense, 24.210-240 Niterói RJ Brasil, Paulo A. M. dos Santos, pams@if.uff.br, Laboratório de Óptica Não Linear \& Aplicada, Instituto de Física, Universidade Federal Fluminense, Niterói RJ Brasil. Os autores agradecem ao MCT/CNPq pelo apoio financeiro recebido através das concessões 3031102/2006-3 e 474218/2006-6.

\section{INTRODUÇÃO}

Cabos ópticos de Telecomunicações são tradicionalmente monitorados quanto às atenuações, reflexões, curvaturas excessivas e descontinuidades de suas fibras usando a conhecida e tradicional técnica de OTDR [1]. Esta última é uma técnica que apesar de poderosa e muito útil, possui limitação em sua sensibilidade. Além do mais, não funciona em tempo real, pois requer a aquisição e promediação de uma grande quantidade de medidas de formatos de onda de retroespalhamento Rayleigh, processo este que pode levar vários minutos, dependendo da extensão do enlace. Isto se deve ao fato de que o sinal retroespalhado está dezenas de dBs abaixo do sinal óptico de varredura, o que produz um formato de onda extremamente ruidoso. A técnica de OTDR não é capaz de detectar distúrbios atuantes no cabo óptico, como por exemplo, aqueles decorrentes de simples contato mecânico nas fibras ou vibrações num determinado local do cabo. Se um intruso quiser acessar fisicamente a uma fibra do cabo ou uma retroescavadeira estiver operando muito próxima do cabo óptico enterrado, este será submetido a esforços transversais e axiais de muito baixa magnitude que o OTDR não será capaz de detectar nem em tempo remoto, exceto, obviamente, se a fibra for rompida ou suficientemente curvada.

Neste trabalho, descreve-se pela primeira vez medidas de resposta no domínio do tempo de um sensor modalmétrico à fibra óptica operando em torno de $1550 \mathrm{~nm}$ sob a ação de perturbações de contato mecânico de muito baixa magnitude. Embora de caráter parcialmente qualitativo, os resultados mostram que a fibra sensora comporta-se como uma espécie de "nervo de vidro" muito sensível aos distúrbios mecânicos de baixa magnitude, que no entanto, não mostraram resposta $\mathrm{DC}$ ou $\mathrm{AC}$ às súbitas variações de temperatura aplicadas pontualmente.

A técnica aqui descrita, embora em estágio de pesquisa experimental básica, tem o potencial de monitorar sensivelmente cabos ópticos de forma a detectar possíveis ameaças a sua integridade física, ou seja, antes mesmo que o dano ocorra, além de localizar a posição do distúrbio. 


\section{EXPERIMENTO E O PRINCÍPIO DE FUNCIONAMENTO DO SISTEMA MODALMÉTRICO}

A Figura 1 esquematiza a configuração experimental utilizada nos testes, assim como ilustra o princípio de funcionamento do dispositivo.

Um laser sintonizável da New Focus capaz de cobrir toda a banda $\mathrm{C}$ emitindo $20 \mathrm{~mW}$ de potência em cada canal foi utilizado como fonte de luz. O sinal em onda contínua é injetado num acoplador SM $2 \times 1$ de $3 \mathrm{~dB}$. O braço de saída do acoplador é emendado por fusão em 3,60 $\mathrm{m}$ de fibra GI-MM onde a mesma é clivada em $90^{\circ}$ na sua extremidade livre. As perturbações são aplicadas pontualmente na fibra MM. A luz é refletida na extremidade, retorna, é re-injetada na fibra SM, passa novamente pelo acoplador e é então detectada. Para isto, utilizou-se um foto-diodo de InGaAs operando em regime fotovoltaico que é acondicionado num receptáculo FC. O sinal elétrico gerado é amplificado com ganho de transimpedância em $10^{5}$ V/A e mostrado num osciloscópio analógico ou digital.

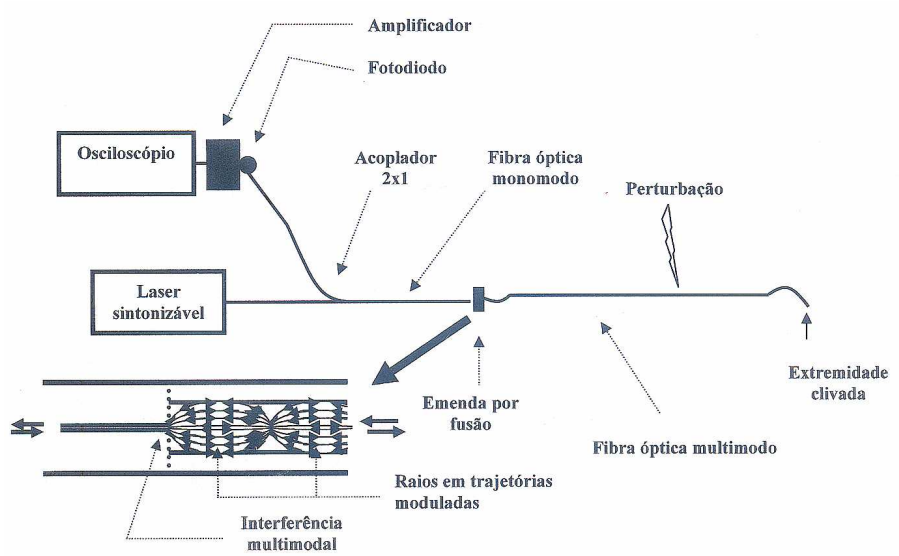

Fig. 1. Configuração experimental do dispositivo. A inserção (em baixo e a esquerda) ilustra o seu princípio de funcionamento.

Diversos mecanismos ópticos de detecção e monitoramento de distúrbios têm sido registrados na literatura [2-5]. A idéia por trás de um sensor modalmétrico aqui apresentado remonta dos anos 80, a partir da emenda por fusão de uma fibra monomodo (SISM) com outra multimodo (SI-MM ou GI-MM), com a finalidade original de realizar medidas de retroespalhamento (OTDR) com filtragem modal. Este procedimento permitiu a caracterização da flutuação de parâmetros físicos de um enlace de fibra GI-MM [6]. Nos anos 90, um sensor modalmétrico foi descrito como um sensor de distúrbios em fibras SM padrão com medidas no domínio das frequiências elétricas [7]. No referido artigo, o mecanismo foi atribuído às interferências ópticas entre os raios que são re-acoplados de volta à fibra SM para o comprimento de onda operacional, que no caso foi visível $(633 \mathrm{~nm})$ [7].
Foi anteriormente demonstrado por um dos autores, um sensor modalmétrico baseado na emenda SI-SM/GI-MM como um sensor pontual de estiramento (strain) operando em $1310 \mathrm{~nm}$ ou $1550 \mathrm{~nm}$ com medidas de intensidade óptica [8]. A seguir, foi investigado o mesmo sensor modalmétrico para estiramento e distúrbios, no domínio do espectro óptico entre 1530-1610 nm [9]. Estes trabalhos [8,9] sugeriram um mecanismo de detecção baseado em interferência multimodal para os distúrbios, e interferência multimodal modulada pela trajetória senoidal dos raios para o estiramento da fibra GI-MM.

A fibra GI-MM foi inventada nos anos 70 [10] com a finalidade de obter um aumento da banda-passante em relação à fibra SI-MM com base no padrão senoidal de trajetórias imposto aos raios propagantes na fibra. Tais trajetórias senoidais com período espacial típico (pitch) de $1 \mathrm{~mm}$, minimizam os atrasos temporais relativos entre os raios. Foi então sugerido um mecanismo de sensoreamento de estiramento (strain) baseado no descasamento entre o período das trajetórias cíclicas e o pitch intrínseco da fibra GI-MM [8]. Quando um estiramento é aplicado na fibra, ocorre uma modulação espacial da fase do grupo de raios (ver inserção da Figura 1) que é convertida em variação de intensidade óptica com período da ordem de $1 \mathrm{~mm}$ quando o sinal é re-injetado na emenda SM/MM. Resultados obtidos sugerem um mecanismo não-interferométrico [8], que são corroborados com medidas experimentais adicionais e um modelo teórico simples [9].

O sensor modalmétrico aqui aplicado apenas para a detecção de distúrbios, gera um sinal de interferência na emenda SM/MM que modula o re-acoplamento na fibra $\mathrm{SM}$, sendo originado pela superposição de $\mathrm{N}$ modos conforme descrito por (1).

$$
\mathrm{I}(\mathrm{r}, \theta)=\sum_{\mathrm{j}=1}^{\mathrm{N}} \sum_{\mathrm{k}=1}^{\mathrm{N}} \sqrt{\left|\mathrm{I}_{\mathrm{j}}(\mathrm{r}, \theta) \mathrm{I}_{\mathrm{k}}(\mathrm{r}, \theta)\right|} \cos \left[\psi_{\mathrm{j}}(\mathrm{r}, \theta)-\psi_{\mathrm{k}}(\mathrm{r}, \theta)+\Delta \Phi_{\mathrm{jk}}\right]
$$

Em (1), j e k designam dois modos quaisquer propagantes na fibra $\mathrm{MM}, \Delta \Phi_{\mathrm{jk}}$ é a fase modificada pela perturbação, enquanto que $\psi_{\mathrm{j}}$ e $\quad \psi_{\mathrm{k}}$ são as fases aleatoriamente distribuídas $\operatorname{dos} \operatorname{modos} \mathrm{j}$ e $\mathrm{k}$, respectivamente [11].

\section{RESULTADOS E DISCUSSÕES}

A Figura 2 mostra a resposta de potência normalizada $\mathrm{P}_{\text {out }} / \mathrm{P}_{\text {in }}$ (em $\mathrm{dB}$ ) do dispositivo como função do comprimento de onda na banda C do DWDM (em torno de $1550 \mathrm{~nm}$ ), ainda sem aplicar qualquer perturbação.

Com referência a Figura 1, o conjunto receptor constituído pelo fotodiodo + amplificador + osciloscópio é substituído por um medidor de potência óptica. 


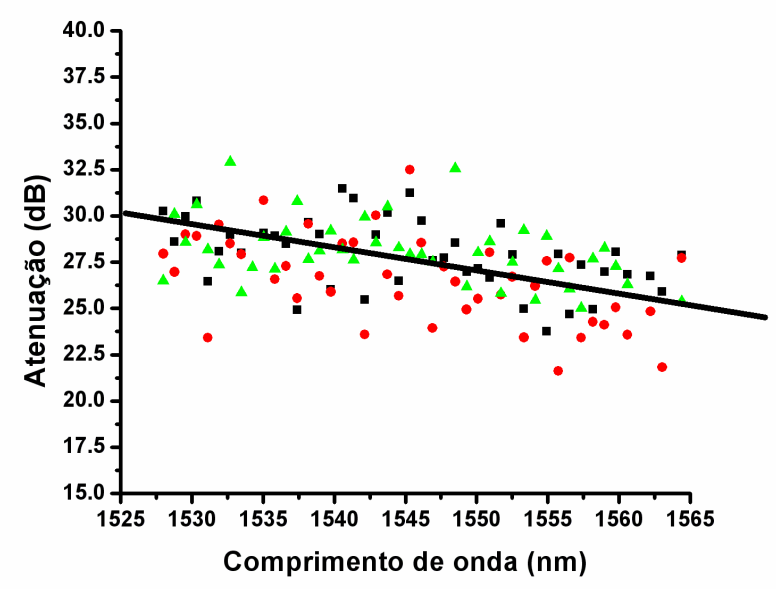

Fig. 2. Resposta espectral estática do dispositivo na banda C, ou seja, entre $1527,99 \mathrm{~nm}$ e $1563,05 \mathrm{~nm}$. A linha contínua é um auxílio visual que permite grosseiramente estimar uma diminuição da atenuação com o aumento do comprimento de onda.

Foram realizadas diversas sequiências de medidas, cada sequência em um dia diferente. O gráfico da Figura 2 mostra a superposição de três destes conjuntos de medidas. Mesmo para apenas uma medida, observou-se instabilidade do sinal de resposta à medida que o comprimento de onda era variado. Em todas as seqüências de medidas, observa-se uma tendência de aumento da atenuação quando o comprimento de onda diminui. No caso, estima-se grosseiramente uma queda média de $\sim 5 \mathrm{~dB}$ ao longo da banda $\mathrm{C}$ (ver no gráfico uma linha contínua de auxílio visual). Estes resultados dispersos mostram a natureza interferométrica (instável) do dispositivo, mesmo sem aplicação de perturbações. A referida instabilidade é então causada pelo próprio meio ambiente, pois o sensor não se encontra isolado de vibrações, sinais acústicos e movimentos de ar.

A seguir, perturbações de contato mecânico de muito baixa magnitude foram aplicadas pontualmente na fibra sensora GI-MM. Tais distúrbios são obtidos com um simples e suave toque de dedo num ponto qualquer da fibra GI-MM. Uma grande quantidade de medidas no domínio do tempo foi realizada. Formatos de onda mostrados no osciloscópio em acoplamento AC foram obtidos e fotografados. As Figuras 3 e 4 mostram quatro amostras de medida do dispositivo operando no canal em $1550,11 \mathrm{~nm}$

Pode-se observar com grande freqüência a ocorrência de um ou dois pares de picos com polaridades opostas. Considerando as amostras da Figura 3, pode-se explicar os dois picos como sendo devidos ao ato de encostar e desencostar o dedo na fibra. Em grande parte das amostras, obteve-se um atraso relativo de $\sim 0,2 \mathrm{~s}$, porém atrasos menores e maiores também foram observados, desde que o ato de encostar e desencostar sejam feitos mais "rapidamente" ou mais "vagarosamente". Apenas um suave toque de dedo (sem desencostá-lo da fibra) produz apenas um pico de resposta temporal, não mostrado aqui.
A ocorrência de mais de um par de picos, conforme mostrado na Figura 4, surge provavelmente quando o toque de dedo na fibra acontece ainda com magnitude fraca, porém suficiente para gerar tênues ondas de choque locais capazes de provocar interferências construtivas e destrutivas que modulam o re-acoplamento na emenda SM/MM

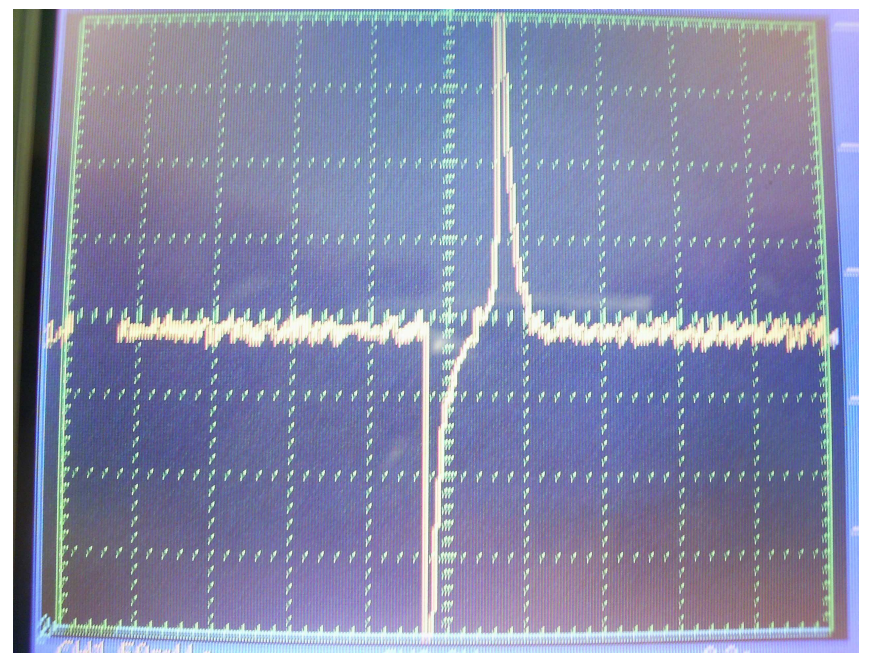

3(a)

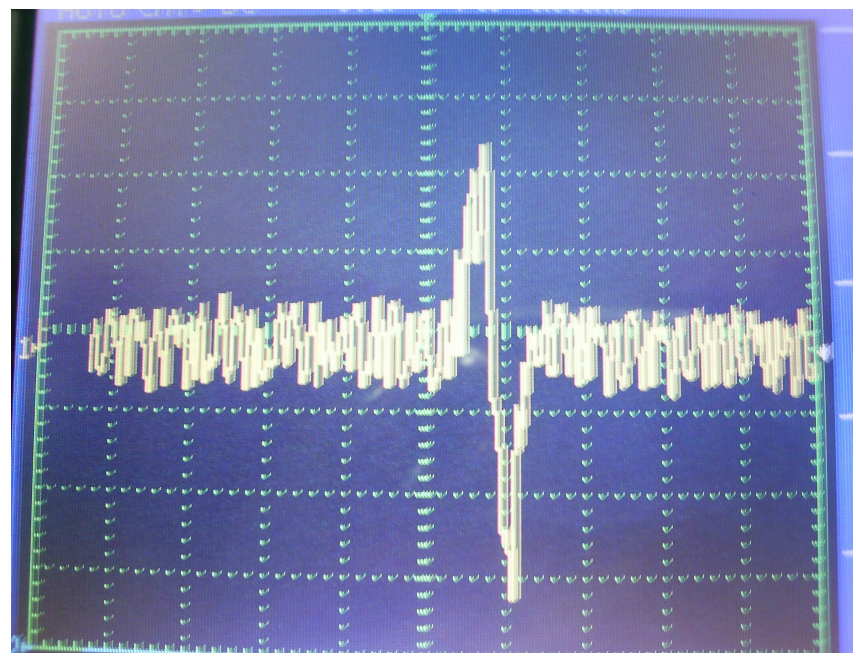

3(b)

Fig. 3. Resposta temporal $(50 \mathrm{mV} / \mathrm{div}$ e $0,2 \mathrm{~s} / \mathrm{div})$ típica consistindo de dois picos de polaridades opostas com (a) $\sim 0,2 \mathrm{~s}$ e (b) $\sim 0,04 \mathrm{~s}$ de atraso relativo.

Quando uma fonte de calor como uma pequena chama, é rapidamente ou lentamente aproximada de um ponto qualquer da fibra MM sensora sem tocá-la, surpreendentemente não foi possível observar qualquer resposta temporal $\mathrm{DC}$ ou $\mathrm{AC}$ na tela do osciloscópio. $\mathrm{O}$ máximo que se observou foram sinais que surgem em decorrência do movimento da fibra devido ao movimento do ar por convecção. 


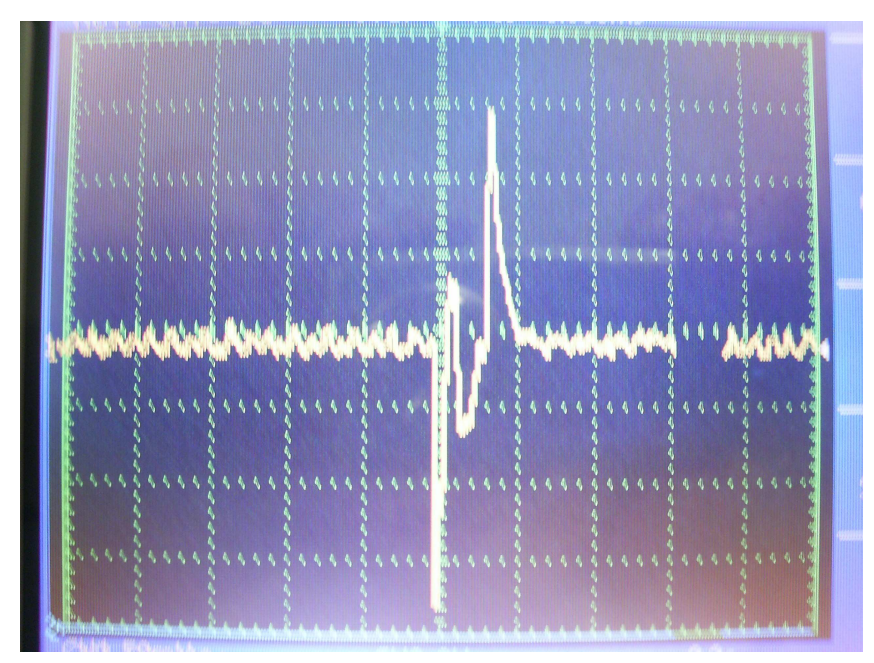

4(a)

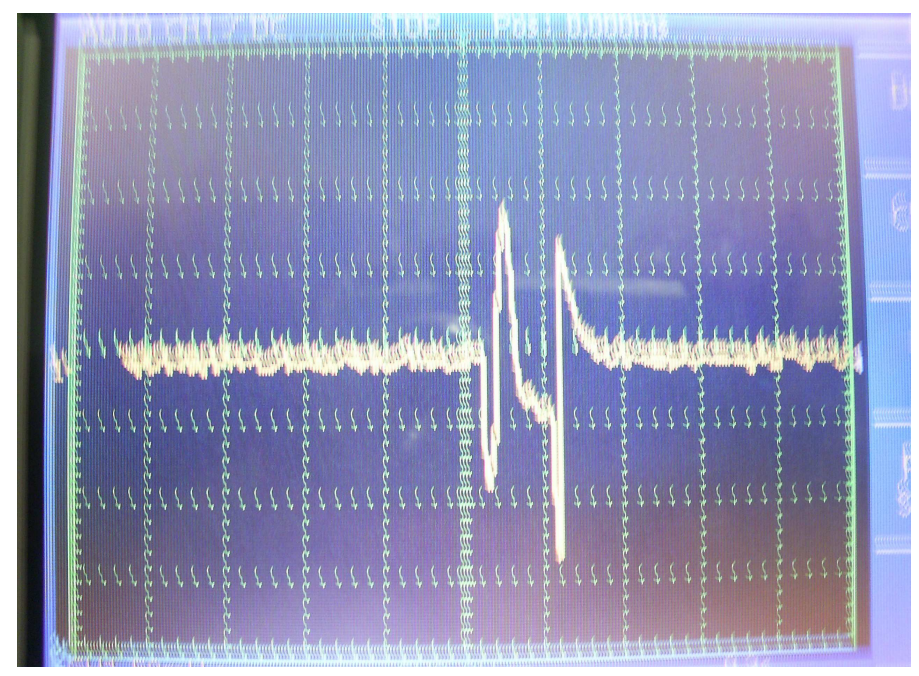

4(b)

Fig. 4. Resposta temporal ( $50 \mathrm{mV} / \mathrm{div}$ e $0,2 \mathrm{~s} / \mathrm{div})$ típica consistindo de quatro picos de polaridades opostas aos pares com $<0,2 \mathrm{~s}$ de atraso relativo.

A Figura 5 mostra a assinatura espectral no domínio óptico do mesmo tipo de distúrbio que vem sendo tratado, porém com um enlace de fibra GI-MM com $1,5 \mathrm{~km}$ de comprimento [9]. Pode-se notar claramente a possibilidade de detecção de um suave toque de dedo quando este procedimento é feito na extremidade remota do enlace. Quando a fibra não é perturbada, observa-se um sinal de baixa amplitude, que lembra ruído. Surge porque na realidade a fibra não está isolada de vibrações, gradientes de temperatura e ondas acústicas originadas do ambiente.

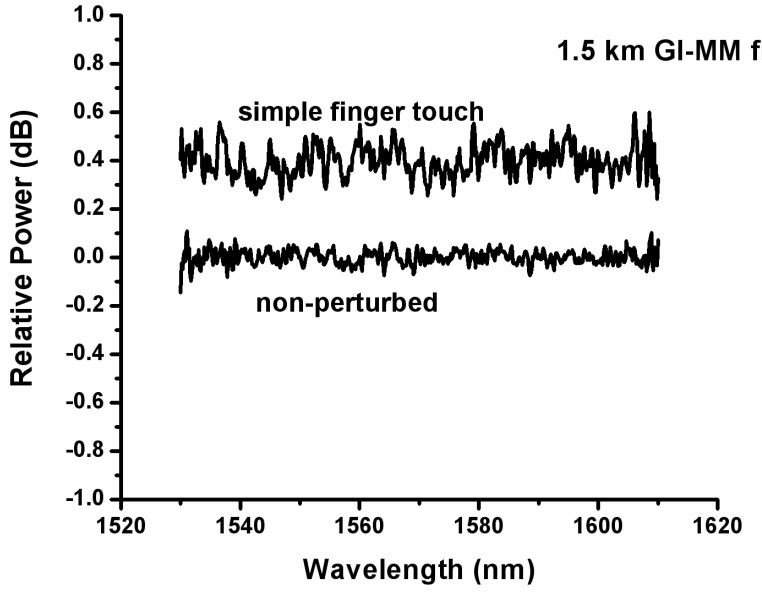

Fig. 5. Resposta espectral no domínio óptico do sensor modalmétrico usando $1,5 \mathrm{~km}$ de fibra GI-MM sensora.

Fibras de sílica utilizadas em Telecomunicações possuem comprimento de onda de corte $\lambda_{\mathrm{C}}$ (cut-off wavelength) tipicamente $>1250 \mathrm{~nm}$ de forma que em 1310 nm e nas bandas $S, C$ e L, estas fibras operem em regime monomodo. Para que a técnica aqui descrita possa ser utilizada, torna-se então necessário que $\lambda_{\text {operacional }}<1250$ $\mathrm{nm}$ o que foi então realizado em $633 \mathrm{~nm}$ num enlace de 6,5 $\mathrm{km}$ com monitoramento da resposta em freqüências elétricas [7]. O sistema fica limitado na distância do enlace quando se faz uso de baixos comprimentos de onda. Como exemplo temos em $1550 \mathrm{~nm}$ cerca de $40 \mathrm{~dB}$ de perda óptica considerando ida e volta num enlace de $100 \mathrm{~km}$ para coeficiente de atenuação de $0,2 \mathrm{~dB} / \mathrm{km}$. Para $850 \mathrm{~nm}$ onde o coeficiente de atenuação é tipicamente $2,5 \mathrm{~dB} / \mathrm{km}$, teríamos uma perda óptica de $250 \mathrm{~dB}$ para $50 \mathrm{~km}$ de enlace. A situação se agrava em $700 \mathrm{~nm}$ onde $\alpha \approx 5 \mathrm{~dB} / \mathrm{km}$ e ainda mais para $\lambda<700 \mathrm{~nm}$ conforme utilizado no enlace em [7] que ficou aparentemente limitado em 6,5 $\mathrm{km}$. Adicionalmente fibras ópticas e componentes passivos em regime monomodo para $\lambda_{\text {operacional }}<1250 \mathrm{~nm}$ são pouco comuns no mercado e exigem núcleos de fibra $<5$ $\mu \mathrm{m}$ o que dificulta o acoplamento de luz.

Se a fibra de Telecomunicações já for MM, normalmente utilizará $850 \mathrm{~nm}$ e/ou $1310 \mathrm{~nm}$ como comprimentos de onda de comunicação. Neste caso é fácil multiplexar e demultiplexar $1550 \mathrm{~nm}$, que se torna o comprimento de onda de operação do monitoramento de distúrbios pelo sistema modalmétrico.

Se a fibra de Telecomunicações for SM, então é possível multiplexar e demultiplexar comprimentos de onda menores que $1250 \mathrm{~nm}$, tipicamente menores que $850 \mathrm{~nm}$ [7], porém o enlace monitorado fica bem limitado na distância. Alternativamente, pode-se instalar uma fibra GIMM em paralelo com a fibra SM de Telecomunicações, na mesma cavidade do cabo óptico, de forma que a primeira se torne apenas uma fibra sensora de distúrbios operando em $1550 \mathrm{~nm}$. 


\section{LOCALIZAÇÂO DOS DISTÚRBIOS}

Além de detectar a ocorrência de perturbações, o sistema deverá também ser capaz de localizar automaticamente o ponto onde acontece cada um destes distúrbios. Um equipamento de OTDR tradicional utiliza pulsos luminosos de pequena duração (ns - $\mu$ s) compatível com o comprimento total do enlace de fibra a ser varrido para localizar os eventos. Basicamente o OTDR é capaz de realizar uma contagem precisa do intervalo de tempo transcorrido entre a emissão de um pulso óptico e o retorno do retroespalhamento e/ou de reflexões.

O sistema modalmétrico aqui descrito, utiliza luz em onda contínua, que entre outras vantagens, é bem mais simples de ser implementado do que o circuito eletrônico de um OTDR. Então, a técnica de localização de eventos perturbativos deverá ser distinta da utilizada no OTDR. Considere $\mathrm{z}=0$ a localização do fotodetector, $\mathrm{z}=\mathrm{z}_{0} \mathrm{O}$ ponto onde um certo distúrbio é aplicado, $\mathrm{L}$ o comprimento total do enlace de fibra, $\mathrm{n}_{\text {eff }} \mathrm{o}$ índice de refração efetivo da fibra para o comprimento de onda operacional e c $=3 \times 10^{8} \mathrm{~m} / \mathrm{s}$. A idéia é que em qualquer ponto da fibra haverá um sinal óptico propagando-se no sentido de sua extremidade clivada, e outro, no sentido contrário, retornando para a emenda SM/MM após ter sido refletido pela referida extremidade. $\mathrm{O}$ sinal viajando em direção a emenda SM/MM será detectado no instante de tempo $t_{1}$, enquanto que o outro sinal será refletido pela extremidade e retornará para a emenda SM/MM, sendo então detectado depois, no instante de tempo $t_{2}$. Portanto, o fotodetector irá capturar os dois sinais geralmente superpostos, mas com um atraso relativo dado por (2).

$$
\Delta \mathrm{t}=\mathrm{t}_{2}-\mathrm{t}_{1}
$$

Com um simples cálculo cinemático, obtém-se a expressão (3), que permite obter a localização $\mathrm{z}_{0}$ onde ocorre o distúrbio em função do $\Delta \mathrm{t}$, desde que o mesmo possa ser precisamente medido e os valores de $L$ e $n_{\text {eff }}$ sejam conhecidos.

$$
\mathrm{z}_{0}=\mathrm{L}-\frac{\mathrm{c} \Delta \mathrm{t}}{2 \mathrm{n}_{\mathrm{eff}}}
$$

No presente caso (ver Figuras 3), onde os sinais apresentam duração total de no mínimo $0,04 \mathrm{~s}$, usando (3) e fazendo $\mathrm{z}_{0}=0$ para a situação mais favorável, conclui-se que seria necessário um enlace de fibra com no mínimo $4.000 \mathrm{~km}$ de comprimento de forma que os sinais possam ser completamente discriminados no tempo e visualizados separados entre si no osciloscópio. A razão é que um processo perturbativo de contato mecânico produz um sinal temporal com duração típica de dezenas de ms ou mais, conforme mostrado pelas Figuras 3 e 4 . Para enlaces usuais ponto-a-ponto onde $\mathrm{L}<100 \mathrm{~km}$, os sinais chegarão superpostos no fotodetector, mas com atraso relativo de $\Delta \mathrm{t}$. Torna-se então necessário processar o sinal composto para descobrir $\Delta \mathrm{t}$, o que implica que o fotodetector combinado com o amplificador deverá ter uma largura de banda compatível e ainda manter o ganho necessário. Considerando uma precisão de $1 \mathrm{~m}$ na localização de um distúrbio pontual, será necessário medir diferenças de tempo da ordem de $5 \mathrm{~ns}$ ao se levar em conta que os sinais ópticos se propagam na fibra com velocidade de aproximadamente $200.000 \mathrm{~km} / \mathrm{s}$. Isto significa que o conjunto fotodetector + amplificador deverá ter resposta temporal (risetime) $<5$ ns mantendo porém o ganho. $\mathrm{O}$ sinal deverá então ser amostrado numa taxa de pelo menos $200 \mathrm{MS} / \mathrm{s}$. Um possível recurso para aliviar a necessidade do uso de um fotodetector e amplificador de grande largura de banda, é inserir uma linha de atraso no enlace, o que pode ser feito emendando alguns quilômetros de fibra GI-MM enrolada numa bobina compacta no final do cabo óptico.

Em primeiro lugar, um sinal de perturbação deverá ser reconhecido frente aos falsos sinais e ruídos, para depois ser possível medir $\Delta \mathrm{t}$. Para a medição (indireta) de $\Delta \mathrm{t}$, deve-se utilizar alguma técnica apropriada de processamento de sinais sobre o sinal composto fotodetectado. Dentre as técnicas conhecidas, faz-se a seguir uma breve discussão sobre a possibilidade de se usar a correlação ou a deconvolução (filltro inverso).

A correlação é uma boa opção quando se tem uma baixa relação sinal-ruído. Usando um detector por correlação, o mesmo conseguiria discriminar o sinal com alta confiabilidade, mesmo que visualmente fosse impossível identificar o sinal na tela do osciloscópio devido ao alto nível de ruído. Detectores por correlação funcionam bem mesmo quando o nível de ruído é mais intenso do que o do próprio sinal a ser detectado. Entretanto, a correlação possui a desvantagem de alargar no tempo o sinal original, o que então deverá dificultar a obtenção de $\Delta \mathrm{t}$.

Uma outra técnica possível é a deconvolução ou filtro inverso [12], bastante similar à correlação mencionada no parágrafo anterior. Uma abordagem consiste em interpretar os dois sinais defasados de $\Delta \mathrm{t}$ e superpostos como a saída de um sistema linear invariante cuja resposta ao impulso $\mathrm{h}(\mathrm{t})$ possui a forma básica de um dos sinais isolados, em cuja entrada aplica-se dois impulsos $\delta\left(t-t_{1}\right)$ e $\delta\left(t-t_{2}\right)$ onde $\mathrm{t}_{1}$ e $\mathrm{t}_{2}$ são os instantes de aplicação dos mesmos com uma separação temporal igual a $\Delta \mathrm{t}$, conforme mostra a equação (2). Desse modo, pode-se construir um sistema (filtro) inverso que produza dois impulsos em sua saída localizados em $t_{1}$ e $t_{2}$, quando o sinal combinado dos dois sinais superpostos for aplicado à sua entrada, obtendo-se assim a separação $\Delta t$ desejada. Este processo é chamado de deconvolução. Uma dificuldade prática é que o filtro inverso exato pode ser instável. Neste caso, pode-se utilizar um filtro pseudo-inverso, que é uma aproximação estável para o filtro inverso. O algoritmo LMS (Least Mean Squares) [13], muito simples, pode ser usado para calcular o filtro pseudo-inverso. Uma vantagem adicional do LMS é que ele vai buscar o filtro pseudo-inverso que maximize a relação sinal-ruído na saída ou uma solução que minimize o erro quadrático entre a resposta obtida e a desejada, o que aumenta a robustez ao ruído (neste caso um bônus). 


\section{CONCLUSÕES}

É aqui descrito o desenvolvimento de um sensor a fibra óptica do tipo modalmétrico para o monitoramento de distúrbios mecânicos. É relativamente bem menos explorado na literatura quando comparado com os demais tipos de sensores ópticos. O foco deste trabalho é aplicar o sistema para o monitoramento da integridade física de cabos ópticos de Telecomunicações, num nível de sensibilidade impossível de se atingir com o uso da tradicional técnica de OTDR. O sistema descrito funciona fazendo acesso a apenas uma das extremidades da fibra sensora. São também propostas e discutidas técnicas que permitam localizar o ponto do cabo onde acontece o distúrbio.

Variando apenas o comprimento de onda $\lambda$ na banda $C$ injetado no dispositivo, obteve-se uma resposta de potência normalizada dispersa em torno de uma média que cresce (ou a atenuação diminui) quando $\lambda$ aumenta. Tal dispersão de valores evidencia a instabilidade do dispositivo devido a sua natureza interferométrica. Uma possível explicação para o fato de que a atenuação diminui com o aumento de $\lambda$ injetado, pode em parte estar relacionado ao aumento do Mode-Field Diameter (MFD) da fibra SM, o que facilita o re-acoplamento de luz na emenda SM/MM [8,9].

Sob comportamento dinâmico, e aplicando uma perturbação bastante fraca num ponto qualquer da fibra MM sensora, observa-se uma resposta em tempo real extremamente sensível no domínio do tempo que não é possível detectar com o uso da tradicional técnica de OTDR. Sinais com amplitude que facilmente pode ultrapassar $200 \mathrm{mV}$ na faixa de até centenas de milissegundos na duração total foram obtidos. Não foi possível observar resposta às súbitas ou lentas variações de temperatura ocorrendo pontualmente bem próximas da fibra MM. O mecanismo de funcionamento do presente dispositivo sensor de distúrbios pode ser explicado como sendo o de um interferômetro de múltiplos raios (ou modos) propagantes ao longo da fibra GI-MM que modula o re-acoplamento na fibra SM.

Sob o ponto de vista de perturbação mecânica, estes resultados são ainda parcialmente qualitativos, pois os distúrbios não são quantificados, mas podem ser compreendidos subjetivamente e facilmente reproduzidos. Atualmente está sendo criada uma técnica para quantificar perturbações de contato mecânico de baixa magnitude, assim como o aperfeiçoamento e a implementação prática da técnica de localização da posição na fibra onde a mesma ocorre, conforme a proposta. Para isto, simulações utilizando o software MatLab estão em curso. A sensibilidade do dispositivo está também sendo aprimorada.

Para finalizar, cabe aqui mencionar que o dispositivo modalmétrico poderá também ter outras aplicações que não sejam a monitoração de cabos de Telecomunicações.

\section{REFERÊNCIAS}

[1] Dennis Derickson, editor, "Fiber Optic Test and Measurement", Prentice Hall, USA (1998).

[2] Alan D. Kearsey, Michael A. Davis, Heather J. Patrick, Michel LeBlanc, K. P. Koo, C. G. Askins, M. A. Putnam and E. Joseph Friebele,

"Fiber Grating Sensors", Journal of Lightwave Technology, 15, 8, 14421463 (1997).

[3] D. Donlagic and M. Zavrsnik, "Fibre-optic microbend sensor structure", Optics Letters, 22, 11, 837-839 (1997).

[4] K. F. Voss and K. H. Wanser, "Fibre optic sensor based upon buckling of a freely suspended length of fibre", US Patent 5,818,982 (1998).

[5] J.C. Juarez, E.W.Maier, K.N. Choi and H.F. Taylor, "Distributed fiber-optic intrusion sensor system", Journal of Lightwave Technology, 23, 6, 2081-2087 (2005).

[6] M. Eriksrud and A. Mickelson, "Application of the backscattering technique to the determination of parameter fluctuations in multimode optical fibres", IEEE Transactions on Microwave Theory and Techniques, vol. MTT-30, 10, 1466-1471 (1982).

[7] I. Oanca, G.Y. Yang, J. Katsifolis and E. Tapanes, "Simultaneous wavelength multiplexed fiber optic communications and cable integrity monitoring technique", CLEO, paper WP4, 106-107, $16^{\text {th }}$ July (1997).

[8] R.M. Ribeiro and M.M. Werneck, "An intrinsic graded-index multimode optical fibre strain-gauge" Sensors and Actuators A, 111, 210215 (2004).

[9] Ricardo M. Ribeiro, "Spectral Response of a Modalmetric Optical Sensor Based on the Graded-Index Multimode Fibre", submetido ao Optics Communications (2008).

[10] D. Gloge and E. A J. Marcatili, "Multimode theory of graded-core fibres", Bell System Technical Journal, 52, 9, 1563-1578 (1973).

[11] Yu A. Bykovskii, O. B. Vitrik and Yu N. Kul'chin, "Amplitude spatial filtering in the processing of signals from single-fiber multimode interferometer, Soviet Journal of Quantum Electronics, 20, 10, 1288-1290 (1990).

[12] Patrick Gaydecki, "A versatile real-time deconvolution DSP system implemented using a time domain inverse filter", Measurement Science \& Technology, 12, 82-88 (2001).

[13] Simon Haykin, "Adaptive Filter Theory”, Prentice-Hall, 1986. 